\title{
Klinkenberg effect in hydrodynamics of gas flow through anisotropic porous materials
}

\author{
Grzegorz Wałowski ${ }^{1, *}$ and Gabriel Filipczak ${ }^{2}$ \\ ${ }^{1}$ Institute of Technology and Life Sciences, Poznan Branch, Department of Renewable Energy Sources, street Biskupińska 67, \\ 60-463 Poznań, Poland \\ ${ }^{2}$ Faculty of Mechanical Engineering, Chemical Engineering Department, Opole University of Technology, street Mikołajczyka 5, \\ 45-001 Opole, Poland
}

\begin{abstract}
This study discusses results of experiments on hydrodynamic assessment of gas flow through backbone (skeletal) porous materials with an anisotropic structure. The research was conducted upon materials of diversified petrographic characteristics, both natural origin (rocky, pumice) and process materials (char and coke). The study was conducted for a variety of hydrodynamic conditions, using air, as well as for nitrogen and carbon dioxide. The basis for assessing hydrodynamics of gas flow through porous material was a gas stream that results from the pressure forcing such flow. The results of measurements indicate a clear impact of the type of material on the gas permeability, and additionally - as a result of their anisotropic internal structure - to a significant effect of the flow direction on the value of gas stream.
\end{abstract}

\section{Introduction}

The gas flow through porous media, both in the aspect of their application in the industrial technology and their presence in the natural environment, is a very complex issue that is not still fully recognised. As for a phenomenological aspect this flow may be subject to various hydrodynamic criteria, which is affected by a medium structure, a kind of fluid (one- and multi-phase) and a flow forcing method (gravitational, pressure). A vast array of publications pertaining to this issue and thoroughly analysed, among others, in studies by Strzelecki [1], Piecuch [2], Orzechowski [3] or Błaszczyk [4] describing this issue on a research and analytical basis de facto refer to the phenomenon of filtration and they are generally identified with the phenomenon of laminar fluid flow through granular deposits according to Darcy's law [5]. Obviously, it does not exhaust many other examples of the flow of fluids through porous media. For the turbulent flow of fluids the Forchheimer model [6] and the Ergun model should be distinguished [7]. The more advanced description of the flow for the spatial layout of capillaries in the form of the meandering channels is also found in the KozenyCarman model [8].

The reference books frequently discuss other models of hydrodynamics of one- and multi-phase fluids flowing through porous media, considering the impact of fluid features and a kind of porous medium on the flow through granular deposits [2-4,9]. However, the vast majority of those models pertain to granular deposits but only a few studies analyse gas permeability through backbone (skeletal) porous materials.
In this context, our own research assesses conditions of hydrodynamics of the gas flow through backbone (skeletal) porous materials with an anisotropic structure. The results of research upon the assessment of gas permeability of various solid porous materials have been presented and the assessment of process conditions concerning hydrodynamics of the gas flow through materials with a diversified internal structure has been conducted.

\section{Scope and research methodology}

To familiarise with hydrodynamic conditions of the gas flow through porous materials, detailed experimental tests were conducted to assess the gas permeability of porous materials with the diversified structure and, at the same time, the diversified process characteristics.

The research material comprised various types of solid skeletal constructions, including those natural and those deriving from the thermal carbon gasification technology. Most of them were coal char in situ, coke, melted waste rock ex situ, and polyamide agglomerate of symmetrical spatial structure.

All the types of materials applied in research underwent the assessment of selected parameters describing features characteristic for porous materials resulting from their porosity and physical structure as basic process quantities affecting the hydrodynamics of the gas flow through porous materials.

The quantity-based assessment applied to such parameters as the apparent density and porosity of a specific type (sample) of the porous material. In this regard, structural research upon the tested porous

\footnotetext{
*Corresponding author: g.walowski@itp.edu.pl
} 
materials conducted on the basis of the SEM scanning image [9] was helpful.

The experiments pertained to two different measurement systems thoroughly analysed in other own works $[10,11]$. The first system was used to assess the permeability of porous materials in the barbotage conditions. In this case, the shape of samples resulted from naturally obtained parts of the native material with an unspecified sample shape - Fig. 1a.

The latter one was applied to analyse the permeability on the basis of the samples configured to the shape of the cubic solid - Fig. 2a. In this system the gas flow might be directed with respect to the arbitrarily selected $\mathrm{X}, \mathrm{Y}$ and $\mathrm{Z}$ axes. This required the development of a special measurement system that is currently being patented [12]. In their geometrical form those cubic-shaped samples were parts of volume samples and were compared with them with respect to their internal structure

Fig. 3 shows some examples of materials used in the experiments. We can see that these materials are very different in their structure, which in addition is anisotropic in every case. Furthermore, for char (Fig. 3a) the slot-porous structure is dominant. Of course, each material has such a different structure in directional cross section $(\mathrm{X}, \mathrm{Y}, \mathrm{Z})$, as for the coke is shown in Fig. 4. a)

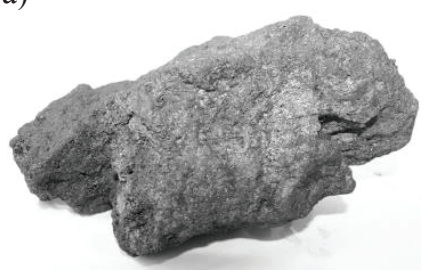

b)

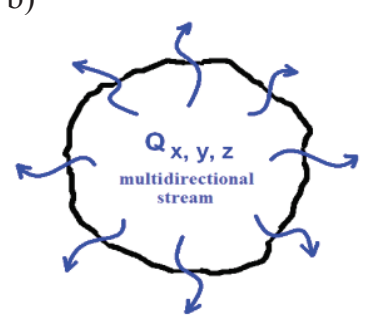

Fig. 1. Sample of unspecified shape: a) research material, b) flow chart (multi-directional - fractal flow). a)

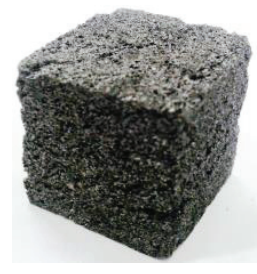

b)

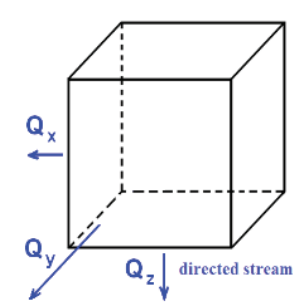

Fig. 2. Cubic-shaped sample: a) research material, b) flow char (X, Y, Z direction flow).

The investigation tests were conducted with reference to various gases (air, nitrogen, carbon dioxide) to the extent of the permeability stream resulting from the reference pressure. The permeability function of the pressure decline in the porous deposit was independently carried out, assuming the so-called multi-directional (fractal) system for the gas flow through samples with unspecified shapes (Fig. 1.b) and the directional flow XYZ characteristic for cubic-shaped samples (Fig. 2.b). a)

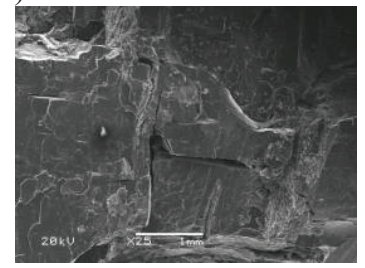

c)

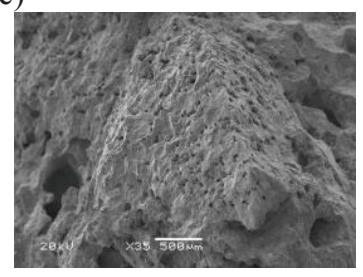

b)

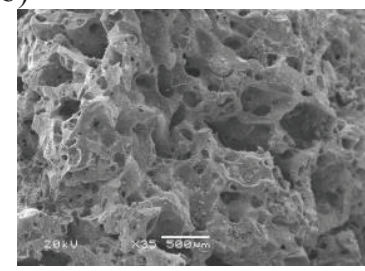

d)

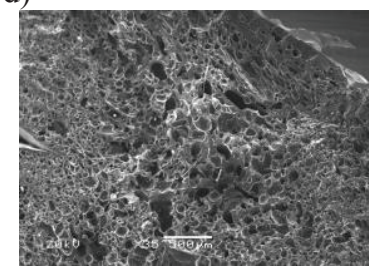

Fig. 3. Structure of tested materials: a) char in situ, b) coke, c) partially melted waste rock, d) char ex situ.

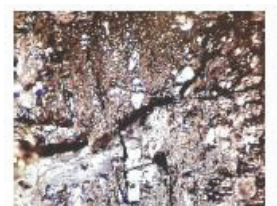

- direction X

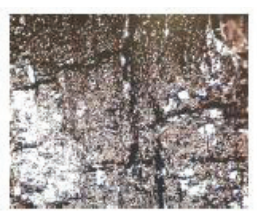

- direction Y

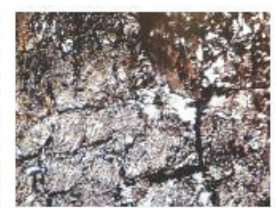

- direction Z
Fig. 4. Sample of coal-char structure in a different direction.

A detailed description of the experimental set-up is given in source work [9], where a description of the test conditions is also provided.

\section{Research results and their analysis}

The basis for assessing the hydrodynamics of the gas flow through deposits and porous materials is the characteristic of their permeability resulting from the pressure inducing this flow. In each case, this characteristic is determined by calculating the impact of the available overpressure on the obtained gas stream or vice versa - the impact of the gas stream on the value of this overpressure that corresponds to a decline in this stream pressure. In the latter case, this corresponds to the determination of complete resistances of the gas flow through such deposit.

The results shown in Fig. 5 prove that with respect to porous materials in the form of coal char the nature of changes to gas permeability functions is highly diversified. For the same coal char in situ (I-1, I-2, I-3) there are obtained highly different permeability characteristics and their common deviation is expressly affected by the structure of the porous material.

Moreover, those characteristics are parabolic, which proves their similarity to the hydrodynamics of the flow through the closed channels. On the other hand, the nonlinear tendency of those characteristics proves the dominance of the turbulent flow, which is also associated with the deviation from Darcy's law $[13,14]$. The comparison to other materials as shown in Fig. 5 shows that coal char in situ (I-1, I-2, I-3) are porous structures which are much more permeable compared to 
coke (II-1, II-2, II-3) or melted waste rock (III-1, III-2, III-3).

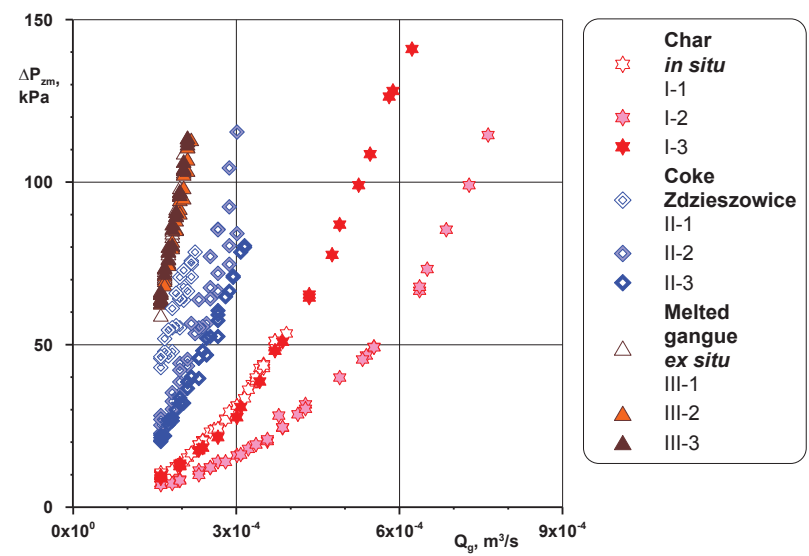

Fig. 5. Permeability of various kinds of porous materials (volume sample); I- coal char in situ, II- coke (Zdzieszowice), III- melted waste rock ex situ.

As for coke which has the highest porosity in this group this proves that a large part of its pores is closed for the gas flow and, concurrently, that the more complex structure of coal chars has characteristics of the gap medium, which at the same reference pressure ensures a much better gas permeability for this medium. On the other hand, in comparison with the aforesaid materials the permeability of the waste rock is undoubtedly justified by a relatively smaller porosity of this material and with the less participation of pores open for the flow [13].

The analogous characteristics of permeability were made for the cubic specimen $(20 \times 20 \times 20 \mathrm{~mm})$ by using the measurement system assessing permeability in the directional flow (Fig. 2). In this measurement, example results of measurements for coal char in situ and sintered polyamide agglomerate are illustrated in Fig. 6, which shows characteristics of the air permeability in three independent flow directions $(\mathrm{X}, \mathrm{Y}, \mathrm{Z})$.

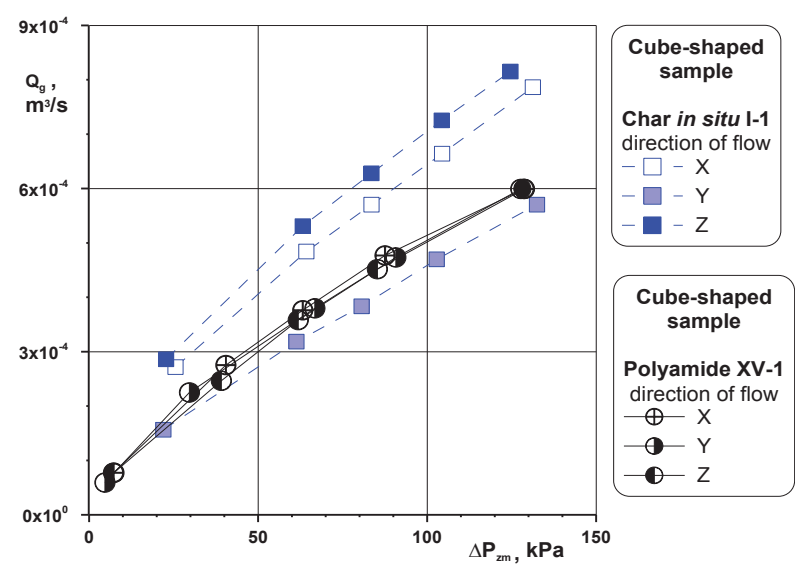

Fig. 6. Layout of experimental points characterizing the asymmetry of air flow with respect to three axes of cube specimen (XYZ) for coal char and sintered polyamide.

The layout of experimental points shows that the permeability of coal char is considerably affected by the direction of the gas flow. This proves the explicit effect of the flow asymmetry with respect to the selected flow direction (axis) and, consequently, the explicit anisotropic structure of this type of material (Fig. 4). On the other hand, for the porous polyamide that forms the agglomerate of spherical particles of identical dimensions (diameter of $0.1 \mathrm{~mm}$ ), the permeability characteristics is not practically affected by the gas flow direction. At the same time, the determined characteristics prove that this porous polyamide, despite the fact its porosity is much smaller compared to coal char $(30 \%$ [9]), has similar characteristics of the gas flow. This proves the observation that the greater effect of the permeability of various kinds of coal char is rather the result of their porous and gap structure rather than the result of their porosity. It is interesting that the permeability characteristic of the porous polyamide is also of non-linear nature, which - with respect to the measurements - proves the advantage of the turbulent gas flow.

At the same time, the research results shown in Fig. 7 prove that the permeability of the porous material is not affected by the sample shape but by its internal structure. The layout of the experimental points shows that in the same conditions of the reference pressure between the volume coal char sample and the cubic-shaped sample (the figure shows averaged air flow values), the permeability characteristics of this material are of a similar nature and within the same scope of values.

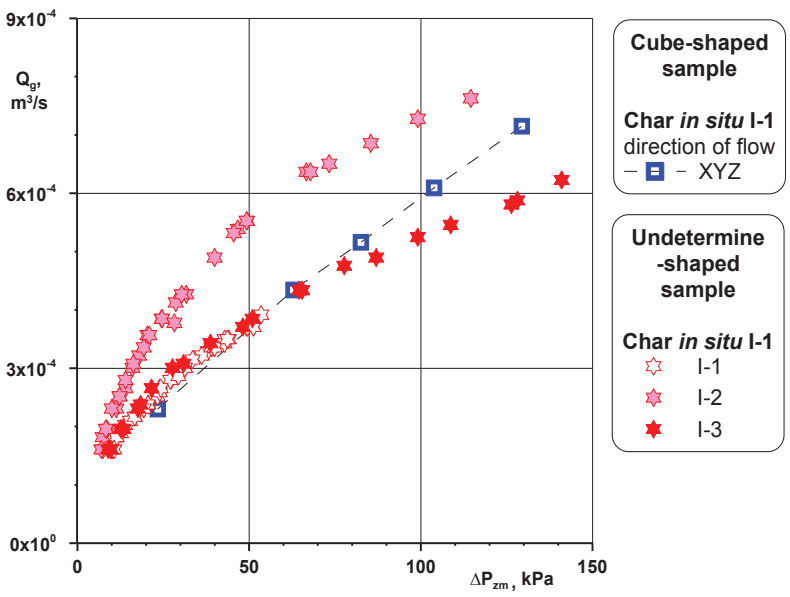

Fig. 7. Gas permeability of coal char for samples of various shapes.

The comparison of permeability characteristics of coal char in situ with respect to the flow of air, nitrogen and carbon dioxide for cubic-shaped specimens (with dimensions of $20 \times 20 \times 20 \mathrm{~mm}$ ) is shown in Fig. 8 .

Regardless of the detected anisotropy of this material, it may also be observed here that to the entire extent of the reference pressure, the permeability of this coal char in situ is less for air than for nitrogen but considerably higher than for carbon dioxide. For this latter gas the limited permeability characteristics was also observed. Undoubtedly, this refers to the choking phenomenon that limits the permeability growth and increases the reference pressure. This may be affected by the fact that the density is higher compared with the remaining ones but according to the fact proven in 
reference books [15] it is more reliable that the hyphenation choking effect expressed by a pressure decline in the porous material is greater, the greater resistance gas flowing the dense network of micro channels encounters (adiathermal conditions). On the other hand, other researchers [16] suggest that this may be affected by the so-called Klinkenberg's effect - as a phenomenon that limits the movement of gas molecules with sizes of flow (pores) channels. Furthermore, this may be due to the different scale of gas expansion when flowing through the microcapillaries. In the latter case, attention is drawn to the significance of this phenomenon, among others, in the aspect of the sequestration of carbon dioxide in geological porous deposits.

On the other hand, the observed differences in permeability for the different flow directions of the gas are undoubtedly the result of a differentiated flow pattern in each direction. This is revealed itself of each different flow direction, different for each direction, as well as the different geometry of the capillaries, the shape and size of which are for flowing gas very different and at the same time which are difficult to assess unequivocally.

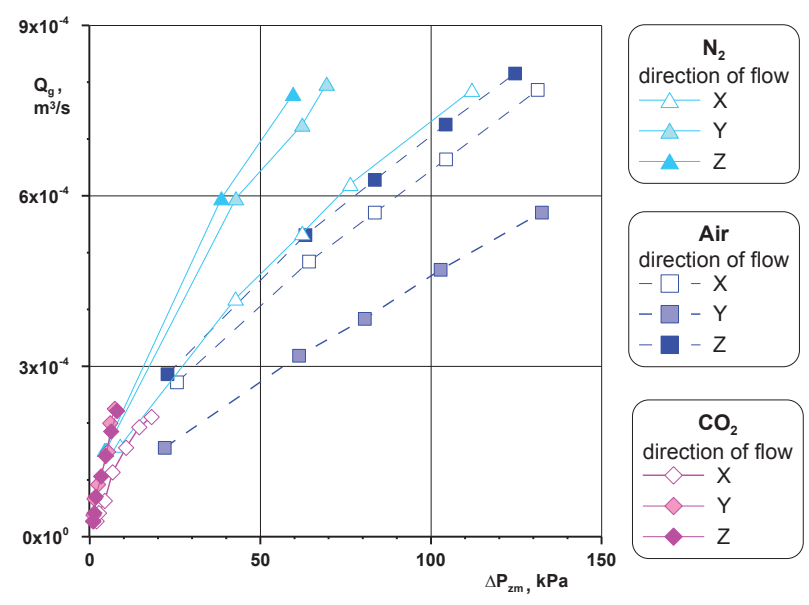

Fig. 8. Permeability of char in situ depending on a working medium (cubic specimen with dimensions of $20 \times 20 \times 20 \mathrm{~mm}$ ).

\section{Conclusions}

The recognition of the issue of hydrodynamics of the gas flow through skeletal porous media shows that the reference books contain very little information. In this respect, relevant experiments on porous materials were conducted and hydrodynamic phenomena assessments resulting from gas flow resistances were carried out.

The hydrodynamic results of gas flow through porous beds with skeletal structure can in many cases be used in the process calculations of hydrodynamics of gas flow through porous beds, especially in the context of biofuel of shale ore [17], biofilm formation by methanogenic microorganisms [18]. Retention in the process of underground bioconversion when selecting strains of microorganisms for maximum biogas yield [19].

The study conducted as part of the project financed by the National Centre for Research and Development conducted in the BIOSTRATEG program, contract No BIOSTRATEG2/298357/8/NCBR/2016 dated 18 May 2016.

\section{References}

1. T. Strzelecki, S. Kostecki, S. Żak, Modelling of flows through porous media (Dolnośląskie Wydawnictwo Edukacyjne, Wrocław, 2008) [in Polish]

2. T. Piecuch, Rocznik Ochrona Środowiska 11 (2009)

3. Z. Orzechowski, J. Prywer, R. Zarzycki, Mechanics of fluids in engineering and environmental protection (WNT, Warszawa, 2009) [in Polish]

4. M. Błaszczyk, Study of migration processes of petroleum substances and their emulsions in porous structures, $\mathrm{PhD}$ dissertation, Dep. of Chemical Eng., Lodz University of Technology, Łódz, 2014 [in Polish]

5. B. Bębenek, Energy losses in fluid flows, (Wydawnictwo Politechniki Krakowskiej, Kraków, 1987) [in Polish]

6. A.M. Amao, Mathematical model for Darcy and Forchheimer flow with applications to well performance analysis, Master's thesis, Department of Petroleum Eng., Texas Tech. University, 2007

7. S. Ergun, Chemical Eng. Prog. 48, 2, 89-94 (1952)

8. Z. Kembłowski Theoretical fundamentals of chemical and process engineering (WNT, Warszawa, 1985) [in Polish]

9. G. Wałowski, Hydrodynamics of gas flow through porous materials, $\mathrm{PhD}$ dissertation, Faculty of Mechanical Engineering, Opole University of Technology, Opole 2015 [in Polish]

10. G. Wałowski, G. Filipczak, Inż. Ap. Chem. 51, 6, 396-397 (2012)

11. G. Wałowski, G. Filipczak, Inż. Ap. Chem. 52, 6, 581-582 (2013)

12. G. Wałowski, G. Filipczak, E. Krause, System for determination the coefficient of gas permeability through porous materials with anisotropic structure, Patent RP 225980, Warszawa 2016.

13. G. Wałowski, G. Filipczak, J. Sustainable Min (2017), http://dx.doi.org/10.1016/j.jsm.2017.03.003

14. G. Wałowski, G. Filipczak, Inż. Ap. Chem. 55, 6, 245-250 (2016)

15. S. Michałowski, K. Wańkowicz, Termodynamika procesowa (WNT, Warszawa, 1993)

16. J. Blicharski, R. Smulski, AGH Drilling Oil Gas Vol. 29 1, 89-96 (2012)

17. C. Erust et al. J. Chem. Technol. Biotechnol. 88, 2115-2132 (2013)

18. D. Chudy, S. Jabłoński, M. Łukaszewicz, Acta Sci. Pol. Biotechnologia, 10, 4, 17-28 (2011)

19. A. Stachowiak i in., Brown coal deposit in the Legnica-Ścinawy region and technologies of their development (Mezozoik i Kenozoik Dolnego Śląska, WIND, Wrocław, 121-135, 2011) [in Polish] 